\title{
Performance of the CMS-HF Calorimeter After Phase I Upgrade
}

\author{
Erhan Gülmez ${ }^{* \dagger}$ \\ On behalf of the CMS-HCAL Collaboration \\ Bogazici University, Bebek, Istanbul, 34342, Turkey \\ E-mail: gulmez@boun. edu.tr
}

CMS-HF Calorimeters have been improved substantially within the Phase I upgrade program. The changes that were finalized during Extended Year End Technical Stop of 2016 - 2017 involved the replacement of the single anode PMTs with the four-anode ones in the HF detector and the associated front-end electronics. Four-anode PMTs were more effective in reducing the noise in the HF detectors due to the window events caused by the muons hitting the PMT windows directly. These PMTs have also thinner windows, further reducing this Cherenkov radiation produced in the windows. Front-end electronic cards were designed to read out the four-anode signals in two channels instead of four to reduce the costs; also, a feature to measure the arrival time of the signals was added. The TDC information is useful to identify the window events that come earlier than the regular signals. Reading the four-anode PMTs with these new cards helped reduce the noise due to the HF in the data collected during the collisions. In this paper, details and the final commissioning of the upgrade will be given. Timing and the comparison of the charge signals from the two channels actually seen in the collision data will be shown.

Sixth Annual Conference on Large Hadron Collider Physics

4-8 June 2018

Bologna, Italy

\footnotetext{
* Speaker.

$\dagger$ This work was supported in part by Turkish Atomic Energy Authority (TAEK) and Bogazici University Research Fund Grant Number: 14040 (18B03P2).
} 


\section{Introduction}

The CMS-HF Calorimeter is designed to detect the particles in the very forward region, $3<$ $\eta<5$, produced by the LHC $p p$ collisions. There are two cylindirically-shaped units placed at each end of the CMS detector. Each unit is $1.65 \mathrm{~m}$ long and has an active radius of $1.4 \mathrm{~m}$. Plasticclad quartz fibers are the active elements. The fibers are embedded in the iron absorber. There are two different length fibers; long $(1.65 \mathrm{~m})$ fibers that are sensitive to both EM and hadronic showers and short $(1.43 \mathrm{~m})$ fibers sensitive to only hadronic showers. Particles produce Cherenkov radiation when they pass through the fibers and this light is carried through the fibers to an air-core light-guide. PMTs are attached to these light guides.

\section{Reason for the Phase I Upgrade: Window Events}

HF Calorimeter operates on the principle that the particles produce Cherenkov radiation in the fibers. The overall charge collected in the PMTs helps us determine the energy of the initial particle producing the shower. However, if a particle, such as a muon, passes through the window of the PMT directly, it produces Cherenkov light right at the window of the PMT, resulting in a very large signal. Such a large signal causes a false trigger for discovery type events. Initially, the correlation between the long and short fiber signals is used to identify the "window events". The anomalous events produce a signal only in one PMT as opposed to the real events that usually produce signals in both the short and long fibers simultaneously. However, as the luminosity increases, so does the number of stray muons in the vicinity of the PMTs making it impossible to eliminate these events, since there will be many uncorrelated window hits in the PMTs attached to both types of fibers.

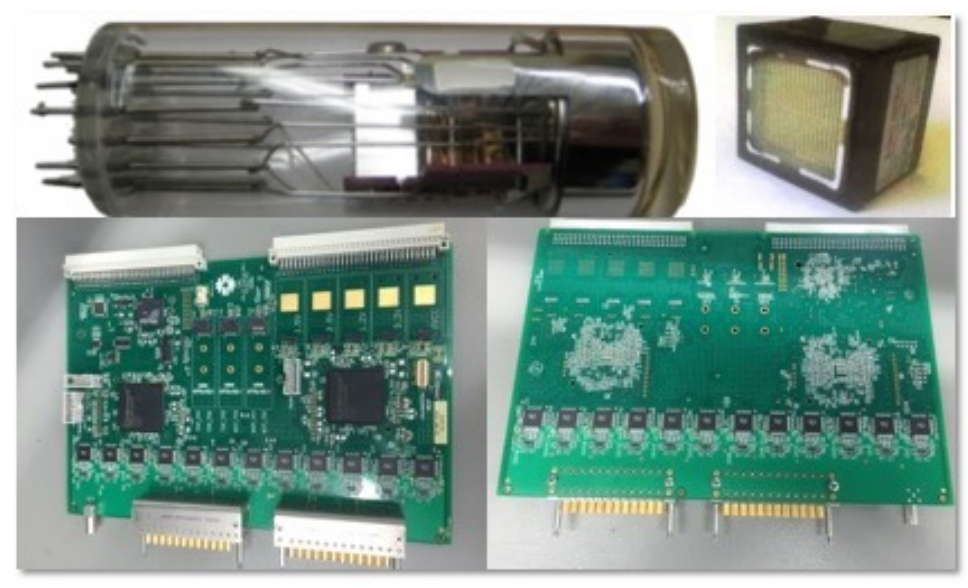

Figure 1: (Top-left) Old PMTs: Hamamatsu R7525; Bialkali photocathode; Max. 25\% quantum efficiency (QE); Typical gain: 5x105; Window: $1.2 \mathrm{~mm}$ thick at the center, $6 \mathrm{~mm}$ thick at the edges; plano-convex geometry with face area $\sim 490 \mathrm{~mm}^{2}$; Glass jacket. (Top-right) Four-anode PMTs: Hamamatsu R7600U200-M4; Ultra Bialkali photocathode; Max. 43\% QE (350 nm); Typical gain: 1.3x106; Window: <1 mm thick; square geometry with area $\sim 324 \mathrm{~mm}^{2}$; Metal jacket. (Bottom row) Pictures of the new HF Front-End Electronic Cards, front and back sides. 


\section{Proposed Solution}

A thinner window four-anode PMT (Fig. 1-top-right) would be a good choice to replace the old single channel PMTs (Fig. 1-top-left). Thin windows result in smaller Cherenkov signals produced on the window glass (Fig. 2-left). Extra anodes would be a powerful tool to eliminate the window events, since the Cherenkov light coming from the real events shines on all the anodes but this is not the case for the Cherenkov light generated at the window [1,2].

Initially, the single channel PMTs in the HF detectors were replaced with the four-anode PMTs during Long Shutdown 1 as part of the Phase I upgrade project. In order to utilize the information coming from the additional anodes, the electronic readout cards were redesigned to handle the PMT signals in two channels, each channel reading a pair of anodes. Furthermore, a TDC feature was also added into these new cards. Window events come earlier than the real events and the timing information provides an additional tool to eliminate them. A nice bonus was that the new four-anode PMTs have higher quantum efficiency almost doubling the signal for the real events (Fig. 2-right).
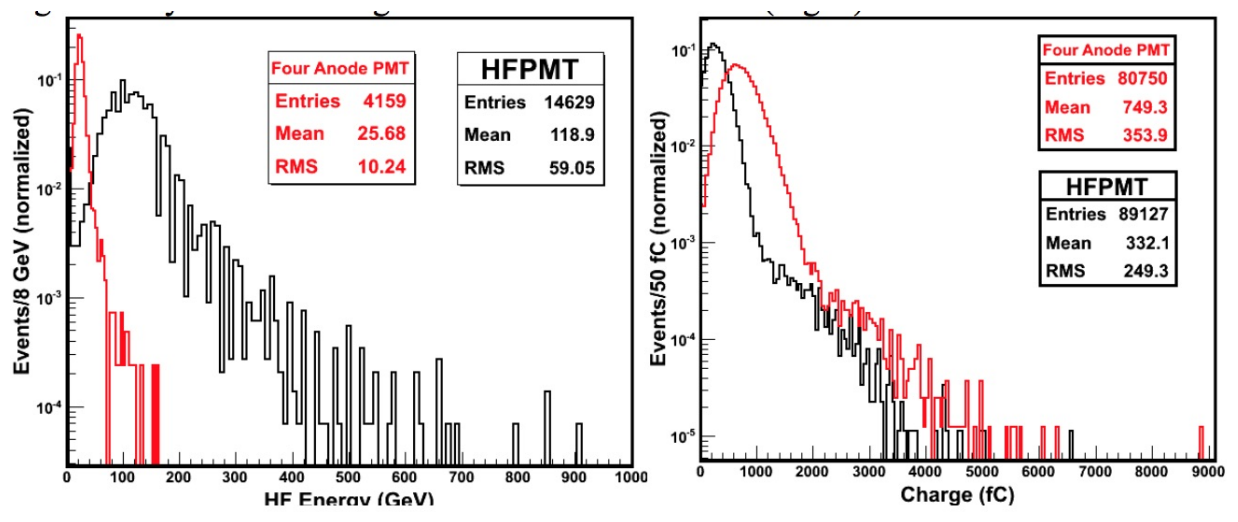

Figure 2: Signals from the old PMTs (black) and new PMTs (red). Pulse-heights are lower for the window events in the new PMTs (left) but higher for the real events (right) [1,2].

\section{Phase I upgrade: Implementation}

New electronic cards are designed to read out the four anodes in two pairs to lower the costs. These two-channel readout cards include the newest version of the ADC chips, QIE10, as opposed to the QIE8 in the previous electronic cards. The new electronic cards also provide information on the arrival time of the signals in each channel in increments of $0.5 \mathrm{~ns}$. Arrival time with respect to the beginning of the ADC integration period provides an independent signature for the anomalous events. As part of the Turkish contribution, 200 cards (Fig. 1-bottom) were assembled in Turkey (by SIMPRO in Istanbul) in 2015 and installed during Extended Year End Technical Stop of 2016 - 2017.

\subsection{HF Front End Electronics Cards and Timing}

Prior to the installation of the new electronic cards, one redesigned card was installed towards 
the end of the 2015 run period. The arrival time of the signals from the PMTs attached to this card was determined by the TDCs in the QIE chip and studied during the 2016 collisions. Cherenkov light coming from the detector travels through the fibers at a lower speed and arrive later than that produced in the PMT window. Two well-separated peaks observed in the TDC spectrum confirm this hypothesis. A two dimensional histogram of the charge collected versus the arrival time of the signals shows that the earlier signals due to the window events are larger than the regular signals as observed in the previous test run results [3] (Fig. 3).

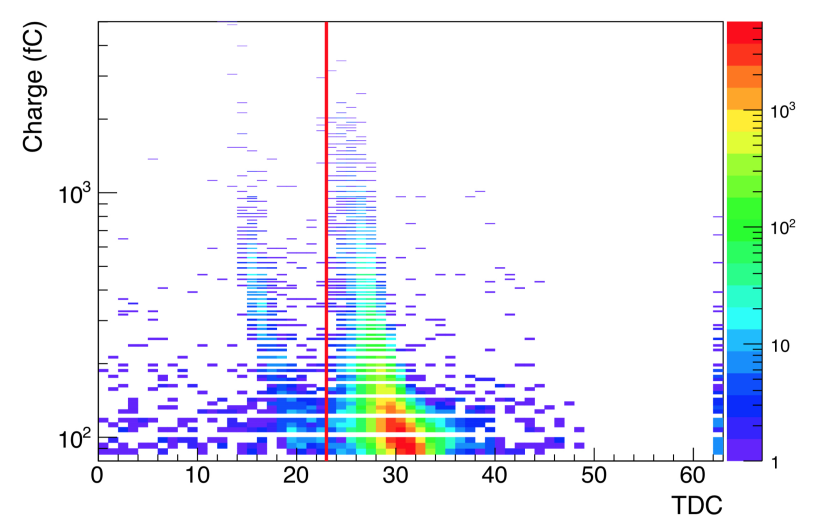

Figure 3: The distribution of the charge collected versus the arrival time of the PMT signals in 2016 collisions. Window events or anomalous events arrive earlier than the regular events (left of the red line) [3].

\section{Performance of the CMS-HF Calorimeter After Phase I Upgrade}

Replacing the old PMTs with the new four-anode ones helped alleviate the anomalous event problem to some extent due to the thinner windows, but it was not enough. With the help of the new two-channel electronic cards, it has become possible to develop algorithms to utilize the additional information coming from the four-anode PMTs. Anomalous or window events tend to produce signals asymmetrically in the PMT, that is, in some of the anodes and not in all of them like the real events do.

The new cards also provide the arrival time of the signals for each channel. It is well established $[1,2]$ that the window events arrive earlier than the regular events. Arrival time versus the charge in a single channel histogram shows that the regular events are around $8 \mathrm{~ns}$ and the window events arrive earlier than 5 ns ((Fig. 4-left) [4].

In addition to the charge versus timing information distribution histogram, Figure 4 also displays the asymmetry plot. Signal asymmetry is calculated as $\left(Q_{1}-Q_{2}\right) /\left(Q_{1}+Q_{2}\right)$ where $Q_{1}$ and $Q_{2}$ are the charge collected in both channels of the same PMT, respectively. Cherenkov light from the detector illuminate the PMT uniformly and produce symmetric signals. On the other hand, the Cherenkov light produced by the window events may not fall on all the anodes equally. As seen in the charge asymmetry plots for the PMTs, the regular events are symmetric between the channels and tend to populate around the center with zero asymmetry. On the other hand, the anomalous events illuminate the PMT partially and produce an asymmetric signal. Events closer to the sides 
or with \pm 1 asymmetry value are window events. The data obtained with the collisions show this nicely as seen in Fig. 4-right [4].
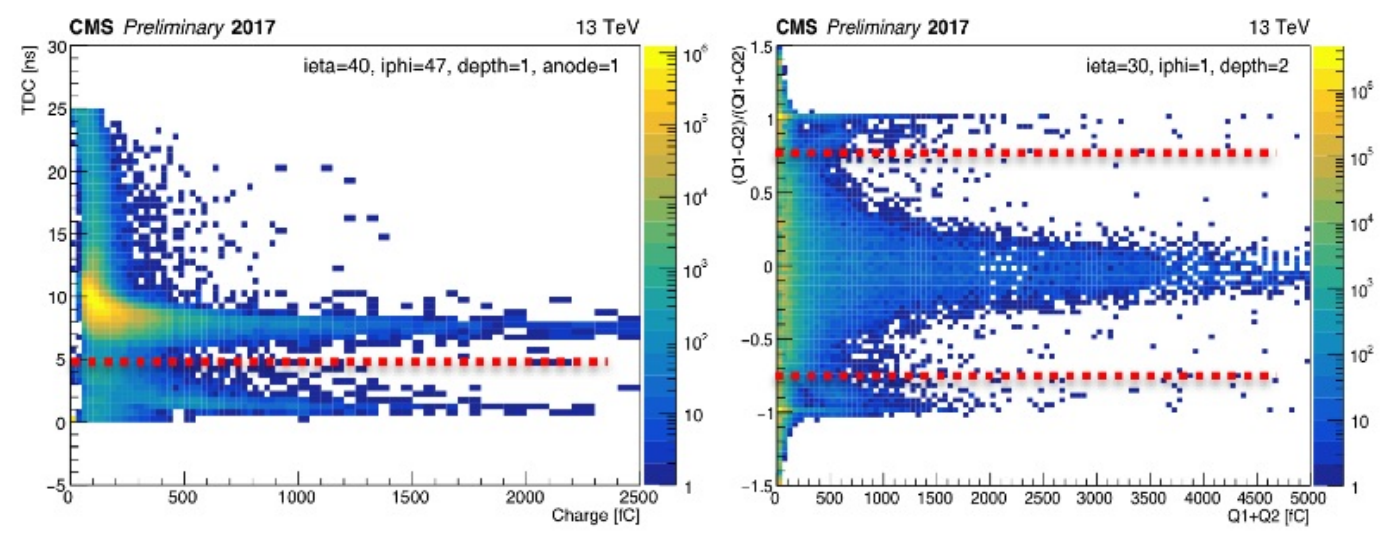

Figure 4: (Left) TDC versus charge in one anode channel in collision data; window events are early ( $<5 \mathrm{~ns})$, real events are around $8 \mathrm{~ns}$. (Right) Charge asymmetry versus total charge in the PMT; signals towards \pm 1 are due to window events, i.e., single channel hits. [4].

\section{Conclusion}

Phase I upgrade of the HF detectors has been completed with the installation and commissioning of the two-channel front-end electronic cards. In addition to the correlation between the electromagnetic and hadronic channels, asymmetry and the timing information provided by the new front-end cards will lead to a higher efficiency in eliminating the unwanted anomalous events and result in a better performance. The new four-anode PMTs are also much better in enhancing the regular signals over the window events.

\section{Acknowledgments}

I would like to thank my colleagues who contributed greatly to the Phase 1 upgrade project and the commissioning of the CMS HF detectors, especially, O. K. Koseyan, B. Bilki, A. Kaminskiy, J. F. Hirschauer, V. Krishtenko and A. Mestvirishvili.

\section{References}

[1] Ph.D. Thesis, Burak Bilki, University of Iowa, unpublished, (2011).

[2] CMS HCAL Collaboration, "Study of various photomultiplier tubes with muon beams and Cherenkov light produced in electron showers," J. of Instrumentation, 5, P06002, (2010).

[3] D. Noonan, "Phase 1 upgrade of the CMS forward hadronic calorimeter," Proceedings ofTopical Workshop on Electronics for Particle Physics, 26âĂŞ30 September 2016, Karlsruhe Institute of Technology (KIT), Karlsruhe, Germany, J. of Instrumentation, 12, C02026, (2017).

[4] CMS Collaboration, "Noise in Phase 1 HF detector in 2017", CMS-DP-2017-034, http://cds.cern.ch/record/2281147, (2017). 